\title{
Yagi-Uda Antenna For L-Band Frequency Range
}

\author{
Gulshan Sharma, Anand N. Sharma, Ashish Duvey, P.K.Singhal
}

Department of Electronics, Madhav Institute of Technology \&Science Gwalior (M.P) 474005-India

Email: gulshan087@gmail.com, ansharma02@gmail.com, a.duvey@yahoo.com, pks_65@yahoo.com

\begin{abstract}
A yagi-uda antenna for L-band $(1-2 \mathrm{GHz})$ frequency range is presented. The designing formulas and related antenna characteristics such as return loss, radiation pattern and gain were also discussed in this paper.
\end{abstract}

Keywords: Proposed yagi-uda antenna, Return loss and antenna beam pattern.

\section{Introduction}

The yagi-uda antenna is basically an arrangement of dipoles in such a way that the whole system provides a directional antenna beam in desired direction. That's why some times it is called a directional antenna system. The yagi antenna's overall basic design consists of a "resonant" fed dipole [1] (the fed dipole is the driven element which are discussed below).

\section{The Elements of Yagi}

\subsection{The driven element}

The driven element of a yagi is the feed point where the feed line is attached from the transmitter to the yagi to perform the transfer the power from the transmitter to the antenna. A dipole driven element will be "resonant"when its electrical length is $1 / 2$ of the wavelength of the frequency applied to its feed point [2]. The feed point is on the center of the driven element. 


\subsection{The directors}

Thedirectors are the shortest of the parasitic element and this end of the yagi is aimed at the receiving station. Itisresonantslightly higher in frequency thanthe driven element, and its length will be about 5\% shorter, progressively than the driven element. The directors length can vary, depending upon the director spacing, the number of directors used in the antenna, the desired pattern, and patternbandwidth and element diameter. Thenumbers of directors that can be used are determined by the physical size (length) of the supporting boom needed by the design. The directors are used to provide the antenna with directional pattern with gain. The amount of gain is directly proportional to the length of the antenna array not by the number of directors used. Thespacing of the directors can range from 0.1 to 0.5 wavelengths or more and will depend largely up $\mathrm{n}$ the design specification of the antenna [3].

\subsection{The reflector}

The reflector is the element that is placed at the rear of the driven element (The dipole).It's resonant frequency is lower, andits length is approximately $5 \%$ longer than the driven element. Its length will vary depending on the spacing and the element diameter. Thespacing of the reflector will be between 0.1 wavelength and 0.25 wavelength [4]. Its spacing will depend upon the gain, bandwidth forward /backwardratio, andside lobe pattern requirements of the final antenna design.

\section{Antenna Design}

For this work, brass wire of $0.1 \mathrm{~mm}$ diameter $(0.1$ is chosen because the ratio of $D / d$ is restricted by wavelength $(\lambda)$, where $D$ is the diameter of boom and $d$ is the diameter of antenna element) is used as antenna element and by using the formula $(\lambda=\mathrm{C} / \mathrm{F})$ the length of the antenna element is calculated and create the separation between the dipoles is $0.1 \lambda$ which is equals to $3 \mathrm{~cm}$. All the physicaldesign consideration for proposed antenna (in $\mathrm{cm}$ )is shown in the figure1.The gain of yagi antenna is depending on the number of dipoles used in the antenna system and each parasitic element provides 3db additional gain. [6] The length and spacing between the elements in terms of wavelength are shown in table1.

Table1: Element length and separation between them in terms of wavelength.

\begin{tabular}{|c|c|c|}
\hline Element & Length & Separation \\
\hline Reflector & $0.55 \lambda$ & $0.1 \lambda$ \\
\hline Driven & $0.50 \lambda$ or $\lambda / 2$ & $0.1 \lambda$ \\
\hline Directors & $0.45 \lambda, 0.40 \lambda, 0.35 \lambda$ & $0.1 \lambda$ \\
\hline
\end{tabular}




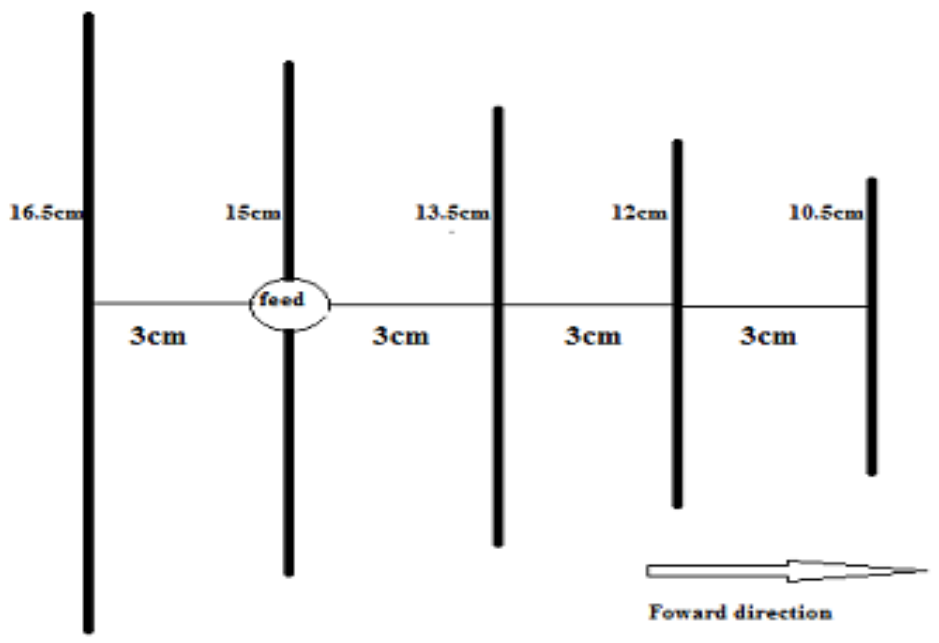

Figure1: Design consideration of proposed yagi-uda antenna.

\section{Measurement and Result}

\subsection{Return loss Measurement}

The measurement is taken by the FS-315 spectrum analyzer connected by SWR bridge and designed yagi antenna. The typical measurement set up is shown in the figure2.We putthe reference level 75 in the spectrum analyzer and all these values are taken in the minimum hold condition to create low difference between actual and measured value

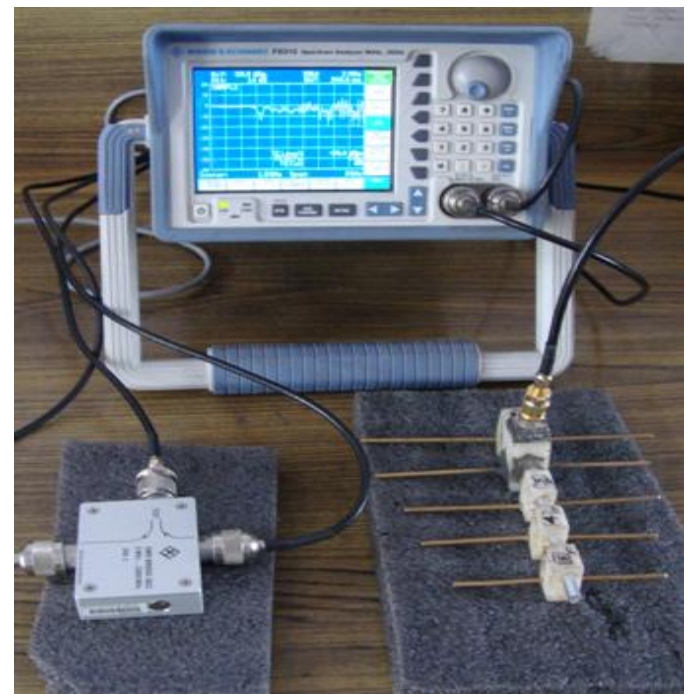

Figure 2: measurement Set-up 


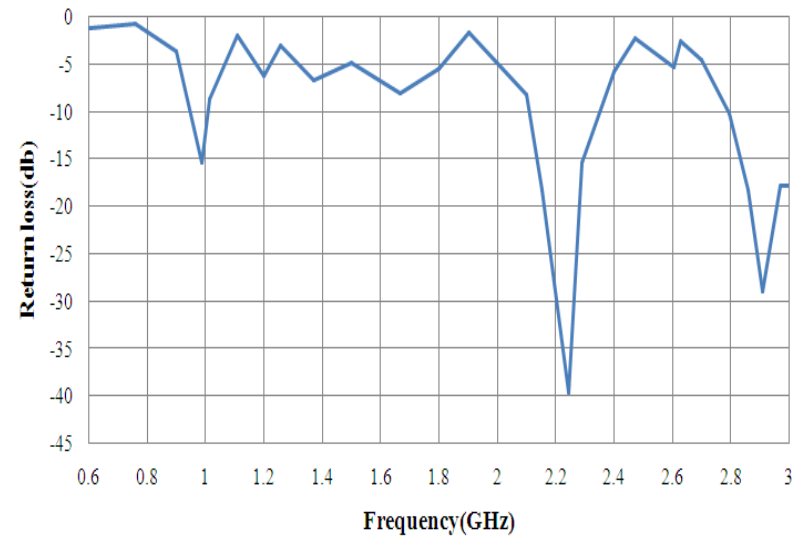

Figure 3: Return loss Vs frequency graph ofproposedyagi-uda antenna.

\subsection{Radiation Pattern Measurement:}

The radiation pattern of proposed yagi-uda antenna(shown in figure 4), is achieved at $1 \mathrm{GHz}$ and to generate the signal of $1 \mathrm{GHz}$ an printed Archimedean spiral antenna have been used which is situated at the transmitting end, and connected to the signal generator.

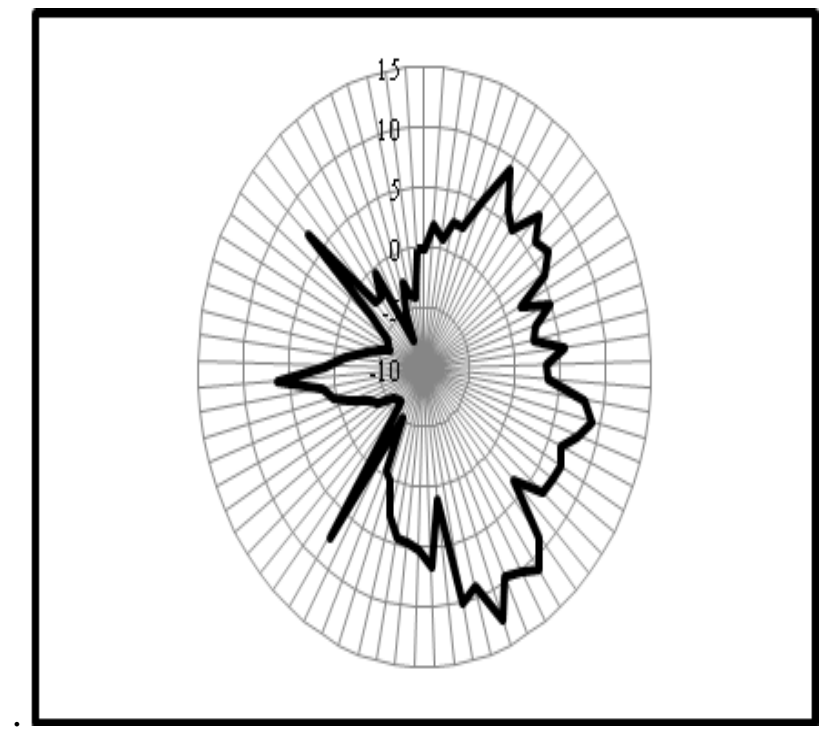

Figure 4:Radiation Pattern of Proposed Yagi-Uda Antenna at $1 \mathrm{GHz}$.

\subsection{Gain measurement:}

Although the theoritical gain of the proposed antenna is about 13-15 db but the measured gain is $9.26 \mathrm{db}$ just because of standard gain antenna which is used as a 
referene gain antenna.Here a microstrip patch antenna with $2.45 \mathrm{db}$ gain is used as a referene gain antenna.

\section{Discussion}

As seen from the figure 3 , it is observed that the proposed antenna successfully works as a multi-frequency yagi-uda radiator and all the radiating frequencies are falls in the range of L-band which is desirable one and as far as the antenna radiation pattern is concerned, the proposed antenna shows directionality (from figure 4) with larger beamwidth which is the basic characteristics of yagi-uda antenna.

\section{Conclusion}

From all the analysis of designed antenna, this paper realized that the designed antenna is successfully works in the L-band frequency range and provides directional antenna radiation pattern with satisfactory gain, which is the object of this paper.

\section{References}

[1] John D. Kraus, "Yagi-uda Antennas" in Antennas second edition1997, page 621.

[2] Sun, B-H, S.G zhou, Y.Fwei, and Q.-Z Liu "Modified two elements" and Yagi-uda“, antenna with tunable beams with tunable beams progress", Progress in Electromagnetic research, vol.16, 406-419,2009.

[3] Balanis, C.A., Antenna Theory, Analysis and Design, 468, 2nd edition, John Wiley \& Sons, Inc., 1997.

[4] Warren L. Stutzman, “Antenna Theory and Design”, Chapter 5.4.Yagi-uda Antenna.

[5] Pozar, D .M., Microwave engineering, 68-70, second edition, Wiley, New York, 1991.

[6] NBS Technical Note 688, tf.nist.gov/timefreq/general/pdf/451.pdf.

[7] GulshanSharma, and P.K. Singhal, "Design of yagi-uda antenna using different shapes of iron rod as antenna element at $2 \mathrm{ghz}$ ", presented at National conference on recent advances in microwave tubes, devices and Communication systems (4-5March, 2011), JNIT- Jaipur NCRA-MTDCS2011.

[8] Densmore, A. and J. Huang, "Microstrip Yagi antenna for mobile satellite service," IEEE Antennas and Propagation Society Int.Symp., Vol. 2, 616619, Jun. 1991. 
[9] Zhang, X. C., J. G. Liang, and J. W. Xie, "The Quasi-Yagi antenna subarrat fed by an orthogonal $\mathrm{T}$ junction," Progress In Electromagnetics Research Letters, Vol. 4, 109-112, 2008.

[10] Chen, C. A. and D. K Cheng, "Optimum element lengths for Yagi-Uda arrays," IEEE Trans. Antennas and Propagation, Vol. 23, Jan. 1975.

[11] Haneishi, M., et al ," Beam-shaping of microstrip antenna by parasitic elements having coaxial stub," Trans. IECE of Japan, Vol. 69-B, 1160-1161, 1986.

[12] Huang, J., Planar microstrip Yagi array antenna,"IEEE Antennas and Propagation Society Int. Symp., Vol. 2, 894-897,Jun. 1989.

[13] Gray, D., J. Lu, and D. Thiel, "Electronically steerable Yagi-Uda microstrip patch antenna array," IEEE Trans. Antennas and Propagation, Vol. 46, No. 5, 605-608, May 1998.

[14] Padhi, S. and M. Bialkowski, "Investigations of an aperture coupled microstrip Yagi antenna using PBG structure," IEEE Antennas and Propagation Society Int. Symp., Vol. 3, 752-755, Jun. 2002. 